\title{
Le fellowship della Società Italiana di Nefrologia: un'intervista
}

\author{
di Elisa Buonanno
}

Nefrologa, Eindhoven - the Netherlands

\begin{abstract}
Italian Nephrology Society (SIN) fellowship: An interview
Abstract. In 2012, the Italian Nephrology Society (SIN), led by President Prof. R. Coppo, established for the first time a fellowship for young nephrologists. Those fellowships are aimed at improving the clinical practice and research skills, through trainings carried out at several Italian nephrology centers. We interviewed Dr. G. Alfano, who spent such a fellowship at a renal ultrasound outpatient clinic to deepen his theoretical and practical knowledge of this instrumental method. His report highlights the importance of fellowships as educational projects and the wish that SIN would provide more fellowships in the future, in order to improve the competence of young nephrologists and to stimulate their professional development.
\end{abstract}

Key words: Fellowship, Italian Nephrology Society (SIN), Young nephrologist

Conflict of interest: None.

Ricevuto: 2 Dicembre, 2012; Accettato: 1 Gennaio 2013

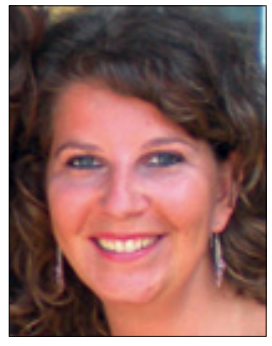

Elisa Buonanno
Nel 2012 la Società Italiana di Nefrologia (SIN), sotto la guida del Presidente Prof.ssa Rosanna Coppo, ha deciso di istituire per la prima volta delle borse di studio rivolte a giovani nefrologi, per finanziare degli stage di attività clinica e di ricerca presso diversi centri di Nefrologia italiani. Le domande pervenute sono state numerose e la SIN, con un notevole sforzo economico, ha premiato tutti i progetti che soddisfacevano $\mathrm{i}$ criteri di selezione indicati.

Si riporta la testimonianza di un vincitore, il dott. Gaetano Alfano, un giovane collega di origini irpine che frequenta il II anno della Scuola di Specializzazione in Nefrologia presso l'Università di Modena e Reggio Emilia, diretta dal Prof. Gianni Cappelli. Nell'ambito della fellowship, il collega ha frequentato lo scorso dicembre l'Ambulatorio di Ecografia Nefrologica presso la S.O.C. di Nefrologia dell'Ospedale "Santa Maria della Misericordia" di Rovigo, diretto dal dott. Fulvio Fiorini.

Gentile Collega, come sei venuto a conoscenza delle fellowship SIN?

Sono venuto a conoscenza, per la prima volta, dell'esistenza della borsa di studio fellowship tramite email inviatami dall'ufficio di segreteria della SIN.
Qual era la tua proposta per il progetto formativo?

Il mio progetto consisteva in un aggiornamento in tema di ecografianefrologicaedecografia vascolareenell'acquisizione di nozioni teoriche di nefrologia interventistica mediante l'attività clinico-ambulatoriale.

La durata dello stage e il supporto economico della SIN sono stati adeguati?

Sia la durata che il supporto economico sono risultati ampiamente adeguati. Lodevole è stato lo sforzo del dott. Fiorini che ha organizzato sedute di ambulatorio ecografico quotidiane; in tal modo sono stati ottimizzati $i$ tempi e si sono raggiunti soddisfacenti livelli di competenza diagnostica.

Che cosa pensi del centro, per cosa ti ha colpito e perché lo consiglieresti (o non) ad altri colleghi?

Durante il tirocinio ho avuto modo di approfondire l'utilizzo degli ultrasuoni nella diagnostica della patologia reno-vescicale, lo studio delle arterie renali e dei grossi vasi arteriosi, la valutazione pre-operatoria degli accessi vascolari ed il monitoraggio delle fistole artero-venose. La mia partecipazione è stata gradualmente sempre più ampia fino al raggiungimento di una sufficiente manualità e di una buona preparazione anatomica-patologica, basi su cui accrescere, in futuro, ulteriori conoscenze. Quindi alla luce delle conoscenze acquisite e dell'ottima ospitalità ricevuta, considero l'esperienza totalmente positiva e stimolante. 


\section{Take-home message della tua esperienza?}

Penso che nella prospettiva di una crescita professionale e di una formazione che promuova il medico nella sua completezza, due risultano $i$ concetti fondamentali: l'approfondimento e il confronto; concetti che hanno trovato, fortunatamente, la loro piena realizzazione nella mia esperienza a Rovigo.

\section{Hai proposte per il futuro?}

Più che una proposta, il mio è un auspicio, affinché la SIN perpetui la sua attenzione e il suo impegno nella formazione dei nefrologi e soprattutto di noi specializzandi, in modo che ciascuno possa utilizzare queste risorse per valorizzare la propria crescita professionale. Inoltre sarebbe davvero interessante che la SIN fornisse indicazioni su tutti quei centri in cui venga promossa una forte attività didattica ed un'attenzione alla divulgazione di conoscenze, centri a cui noi giovani nefrologi potremmo fare riferimento.

Oltre all'iniziativa delle fellowship, negli ultimi anni la SIN sta dando un importante supporto ai giovani nefrologi, per favorirne la partecipazione attiva e la formazione mediante numerose iniziative: dall'iscrizione gratuita alla società all'accesso on-line gratuito a riviste nazionali ed internazionali, dal portale cerco-trovo lavoro ai grant per la ricerca. La testimonianza del dott. Alfano mette in evidenza il valore formativo delle fellowship quali progetti di ricerca fruibili nella caleidoscopica realtà nefrologica italiana, ricca di centri di eccellenza sia universitari che ospedalieri, capaci di entusiasmare e di fornire occasioni di approfondimento e di crescita professionale ai giovani medici.

\section{Riassunto}

Nel 2012 la Società Italiana di Nefrologia (SIN), sotto la guida del Presidente Prof.ssa R. Coppo, ha istituito per la prima volta delle borse di studio dedicate ai giovani nefrologi, finalizzate allo svolgimento di attività cliniche e di ricerca mediante stage presso numerosi centri di Nefrologia italiani. Si riporta la testimonianza di un vincitore, il dott. G. Alfano, che ha frequentato un ambulatorio di ecografia nefrologica per approfondire tale metodica. La sua testimonianza sottolinea l'importanza delle fellowship come progetti formativi e l'auspicio che la SIN ne elargisca sempre di più, per migliorare la preparazione e la crescita professionale dei giovani nefrologi.

Parole chiave: Fellowship, SIN, Giovani medici

Dichiarazione di conflitto di interessi: L'Autore dichiara di non avere conflitto di interessi.

Indirizzo dell'Autore:

Dr.ssa Elisa Buonanno

Geldropseweg 30-30

5611 SJ

Eindhoven, the Netherlands

eli.buonanno@gmail.com 